\section{Physicochemical characteristics and supraliminal odor intensity: Reply to Mitchell}

\author{
WILLIAM S. CAIN* \\ John B. Pierce Foundation Laboratory and Yale University School of Medicine \\ New Haven, Connecticut 06519
}

The rate of growth (exponent) of the psychophysical function for odor intensity depends upon the physicochemical properties of odorous substances. Although no single property is able to predict rate of growth, a model developed by Dravnieks and Laffort that combines three basic properties has been quite successful. The results of intra- $\mathrm{O}$ comparisons of the relative exponents for various odorants have withstood the test of replication, i.e., variation among $O$ s has usually not been a serious problem. In the future, comparisons of the exponents obtained from one laboratory to another would be aided considerably by inclusion of a standard odorant in all scaling studies.

Cain (1969) reported a series of experiments that aimed primarily to examine the possible correlation between the number of carbon atoms in the molecule of an aliphatic alcohol and the exponent of the psychophysical power function for odor intensity. The first experimient dealt with four alcohols and revealed a perfect (negative) rank-order relation between carbon chain length and size of the exponent. This experiment replicated and extended an earlier observation that n-propanol has a sizably greater exponent than n-octanol (Engen, 1965). Three other experiments in the series replicated and extended further the trend found in the first experiment. Five experiments, therefore, led directly to the conclusion that, for the primary aliphatic alcohols, there is a reliable correlation between the exponent and physicochemical properties. For additional experimental evidence, see Rovee (1969), Cain and Engen (1969), Cain (1970), Dravnieks and Laffort (1970), and Berglund, Berglund, Engen, and Ekman (1971).

Cain (1969) also obtained psychophysical functions for two odorants outside the aliphatic series of alcohols. These odorants (acetone and geraniol) were chosen on the basis of solubility in water, a property implicated frequently in the process of olfactory stimulation (cf. Dravnieks, 1967). Water solubility is one of the physicochemical properties that varies monotonically as a function of chain length in aliphatic alcohols, and an inspection of six functions obtained by Jones (1958) suggested that water solubility might bear a general relationship to the size of the exponent. When the psychophysical functions for acetone and geraniol were compared directly to

*Address: John B. Pierce Foundation Laboratory, 290 Congress Avenue, New Haven, Connecticut 06519 . functions obtained for aliphatic alcohols in the same experiment (cf. Experiments 4 and 5), the results were entirely consistent with the notion that the exponent bore a strong relation to water solubility. Nevertheless, the conclusion on this matter was conservative: "The physicochemical properties of odorous substances are often interdependent and may exert their biological effects in concert. Thus, it is important to proceed with caution in any attempt to relate psychophysical properties with a specific physicochemical property. For now, it seems appropriate to conclude that the rate of growth of suprathreshold odor intensity is at least partially dependent on the solubility characteristics of odorants [Cain, 1969, p. 353]."

This conclusion was dictated by the knowledge (1) that thousands of substances are odorous, and a relation obtained with one battery of odorants may not be obtained with another, and (2) that water solubility is only one aspect of an odorant's behavior in a liquid medium. Correlated aspects include, for example, solubility in various oils and lipids and in water-oil solvents. Furthermore, solubility in water is a "phenotypical" characteristic that can be predicted by more basic but less obvious physicochemical characteristics.

\section{PREDICTION OF THE EXPONENT}

Olfactory science has seen little success in efforts to find general relations between particular physicochemical and psychophysical properties. Models to predict relative threshold values, for example, have found success only when they have incorporated more than one physicochemical variable (cf. Davies \& Taylor, 1959; Laffort, 1969). It is not surprising, therefore, to find that the search for the physicochemical correlates of the exponent must, in the light of new data, be broadened to include properties other than water solubility.
Laffort (1970) and Dravnieks and Laffort (1970) have developed a model that successfully predicts the relative steepness of the psychophysical function for odor intensity. This model is derived from three physicochemical indices: (1) molar volume at the boiling point, (2) ability to accept hydrogen bonding protons, and (3) an index of electronic polarizability. An interesting feature of the model is that it seems to show how the relative distribution of odorous molecules into aqueous mucus and onto proteinous olf a ctory receptors varies as a function of the number of odorous molecules at the mucus-receptor interface. It predicts the relative size of the exponent from the ratio of what Laffort" calls "efficient solubility in water" (a factor that reflects hydrogen bonding and electronic polarizability) to the logarithm of the threshold concentration. So far, predictions from the model have been found to correlate significantly with the relative steepness of functions obtained by $\mathrm{Katz}$ and Talbert (1930), by Cain (1969), and by Dravnieks and Laffort (1970), themselves. It will be interesting to learn if the model will be equally successful on the data of Berglund et al (1971).

\section{VARIABILITY OF THE EXPONENT}

As noted above, Cain's conclusions were based upon a number of replications of a trend originally reported by Engen (1965). Mitchell (1971) has argued that the absence of statistical analysis impairs the credibility of those conclusions. To support his argument, Mitchell said: "He [Cain] stated that certain of his data points 'exhibited excessive variability,' and that there was "variability in the exponents yielded by individual Os.' This being the case, it would have seemed incumbent on Cain to display the robustness of his assertions by some statistical justification [p. 477]." Although it is arguable on general grounds that statistical indices (e.g., the standard deviation) can be valuable additions to an experimental report, Mitchell's use of Cain's statements are likely to mislead the reader. The phrase "exhibited excessive variability [Cain, 1969 , p. 350]" referred to two matches of odor intensity and clearly was not intended to apply to the other 116 data points reported. Moreover, the comment about variation in the exponent for individual Os (p. 352) was meant to illustrate a general finding (e.g., Reynolds \& Stevens, 1960; Marks \& Stevens, 1966) that, although the absolute size of an exponent may vary from one group of $\mathrm{Os}$ to another, the relative sizes of the exponents obtained for different substances from a single group of Os are rather stable. Such intra-O comparisons of the relative size of the 
Table 1

Exponents of the Psychophysical Functions for Individual Observers

\begin{tabular}{|c|c|c|}
\hline $\mathbf{0}$ & $\mathrm{N}$-propanol & Geraniol \\
\hline 1 & 1.03 & .28 \\
\hline 2 & .99 & .16 \\
\hline 3 & .61 & .20 \\
\hline 4 & .78 & .70 \\
\hline 5 & .68 & .38 \\
\hline 6 & .51 & .20 \\
\hline 7 & .64 & .19 \\
\hline 8 & .68 & .17 \\
\hline 9 & .89 & .51 \\
\hline 10 & .55 & .54 \\
\hline 11 & .24 & .14 \\
\hline 12 & .59 & .15 \\
\hline 13 & .93 & .21 \\
\hline 14 & .67 & .21 \\
\hline 15 & .47 & .14 \\
\hline Mean & .69 & .28 \\
\hline SD & .21 & .17 \\
\hline SDDIFF & \multicolumn{2}{|c|}{.23} \\
\hline$S E_{D I F F}$ & \multicolumn{2}{|c|}{.06} \\
\hline
\end{tabular}

exponent, as well as the clear correlation between relative size and physicochemical properties, formed the basis of the conclusion that there are reliable differences among the exponents yielded by various odorants.

The standard deviation of the mean exponent obtained from a group of Os varies somewhat with manner of stimulus presentation and appears to differ from one laboratory to another. Berglund et al (1971), who varied concentration by means of liquid dilution, obtained an average standard deviation of 0.15 . This is almost identical to the value Cain (1969) found (but did not report) for odorants diluted with a liquid solvent. For stimuli delivered via an air-dilution olfactometer (Experiment 5), the average standard deviation was 0.21. Mitchell (1971) reports that, with olfactometric stimulus presentation, the typical standard deviation obtained in his laboratory is greater than 0.30 and has been as high as 0.39 . A possible contribution to this high variability may be the small physical range (about 10:1) used by Mitchell and his colleagues. In their experiments, some steps between adjacent stimulus levels appear small enough to challenge the Os' capacity to discriminate successfully one level from another (cf. Gregson, Mitchell, Simmonds, \& Wells, 1969). When an experiment is set up in such a way as to allow estimates of perceived magnitude to be controlled by discriminal capacity, Os may sometimes exaggerate the differences among their estimates in order to demonstrate their ability to resolve small differences among the stimuli (Stevens, 1958), whereas at other times, they may not even be able or may not attempt to resolve small differences.

Table 1 shows exponents yiclded by 15 Os for two odorants, n-propanol and geraniol (cf. Cain, 1969, Experiment 5). This pair of odorants is analogous to the pair (n-propanol and eugenol) studied by Mitchell and Gregson (cited by Mitchell, 1971). ${ }^{2}$ Although the distributions of the exponents for propanol and geraniol overlap, every $O$ yielded a higher exponent for propanol than for geraniol. For this and for similar comparisons, the variability of the differences is more relevant than the variability of the mean exponent. An unbiased estimate of the standard error of the differences for propanol and geraniol is 0.06 , a value quite small compared to the difference between the means. It would be desirable, of course, to find even smaller values for the standard error. Nevertheless, it is noteworthy that a much more modest difference in average exponent than is shown here would reveal itself as statistically significant. It may be also worthwhile to reemphasize that differences due to chance rarely survive the test of replication (cf. Békésy, 1967, p. 111).

Much remains to be done to specify and to minimize the sources of variation in experiments in olfactory psychophysics. Furthermore, until the dependence of the exponent on method of stimulus presentation is more thoroughly explored and until the effects of "regression" that arise from the use of estimation techniques (e.g., magnitude estimation) are counterbalanced by production techniques (e.g., magnitude production), the absolute size of the exponent for any particular odorant will remain to be pinpointed. However, the relative size of the exponent from one substance to another can still be frutfully investigated. Undoubtedly, the dependence of relative size on physicochemical properties will be understood thoroughly only after many odorants have been studied. To facilitate progress in this area, it would be desirable to make the relative values comparable from one laboratory to another. One way this could be done is to include a standard odorant (e.g., n-butanol) in all scaling studies. Such a standard would be analogous to the $1,000-\mathrm{Hz}$ tone in psychoacoustics. Ideally, the standard odorant should be scaled in the same experimental session with the odorant under study. A mixed series of stimuli that included various concentrations of both odorants would allow for the direct comparison of exponents obtained by magnitude estimation.

\section{REFERENCES}

BEKESY, G. von, Sensory inhibition. Princeton: Princeton University, 1967.

BERGLUND, B., BERGLUND, U., ENGEN, T., \& EKMAN, G. Individual psychophysical functions for 28 odorants. Perception \& Psychophysics, 1971, 9, 379-384.

CAIN, W. S. Odor intensity: Differences in the exponent of the psychophysical function. Perception \& Psychophysics, 1969, 6, 349-354.

CAIN, W. S. Odor intensity after self-adaptation and cross-adaptation. Perception \& Psychophysics, 1970, 7, 271-275.

CAIN, W. S., \& ENGEN, T. Olfactory adaptation and the scaling of odor intensity. In $C$. Pfaffmann (Ed.), Olfaction and taste III. New York: Rockefeller University, 1969. Pp. 127-141.

DAVIES, J. T., \& TAYLOR, F. H. The role of adsorption and molecular morphology in olfaction: The calculation of olfactory thresholds. Biological Bulletin, 1959, 117, 222-238.

DRAVNIEKS, A. Properties of receptors through molecular parameters of odorivectors. In T. Hay ashi (Ed.), Olfaction and taste II. London: Pergamon, 1967. Pp. 89-108.

DRAVNIEKS, A., \& LAFFORT, P. Odor intensity function in adult humans: Physicochemical correlates of slope for single odorants. Unpublished manuscript, IIT Research Institute, 1970.

ENGEN, T. Psychophysical analysis of the odor intensity of homologous alcohols. Journal of Experimental Psychology, 1965, 70, 611-616. GREGSON, R. A. M., MITCHELL, M. J., SIMMONDS, M. B., \& WELLS, J. E. Relative olfactory intensity perception as mediated by ratio-range category scale responses. Perception \& Psychophysics, 1969, 6, 133-136.

JONES, F. N. Scales of subjective intensity for odors of diverse chemical nature. American Journal of Psychology, 1958, 71, 305-310.

KATZ, S. H., \& TALBERT, E. J. Intensities of odors and irritating effects of warning agents for inflammable and poisonous gases. Technical Paper 480, Bureau of Mines, U.S. Department of Commerce, 1930.

LAFFORT, P. A linear relationship between olfactory effectiveness and identified molecular characteristics, extended to fifty pure substances. In C. Pfaffmann (Ed.), Olfaction and taste III. New York: Rockefeller University, 1969. Pp. 150-157.

LAFFORT, P. The prediction of olfactory thresholds and slopes of odor intensity. Unpublished manuscript, IIT Research Institute, 1970.

MARKS, L. E., \& STEVENS, J. C. Individual brightness functions. Perception \& Psychophysics, 1966, 1, 17-24.

MITCHELL, M. J. Olfactory power law exponents and water solubility of odorants: $\mathrm{A}$ note on Cain's (1969) study. Perception \& Psychophysics, 1971, 9, 477.

REYNOLDS, G. S., \& STEVENS, S. S. Binaural summation of loudness. Journal of the Acoustical Society of America, 1960, 32, 1337-1344.

ROVEE, C. K. Psychophysical scaling of olfactory response to the aliphatic alcohols in human neonates. Journal of Experimental Child Psychology, 1969, 7, 245-254.

STEVENS, J. C. Stimulus spacing and the judgment of loudness. Journal of Experimental Psychology, 1958, 56, 246-250.

\section{NOTES}

1. P. Laffort. Personal communication. 1970. 2. At the time this paper is being written, three of M. J. Mitchell's papers (two by Mitchell and R. A. M. Gregson and one by Mitchell and R. L. McBride) are "in press" and unavailable to this author.

(Accepted for publication September 14, 1970.) 